\title{
Effects of a Rivastigmine Patch on Self-Care Activities in Patients with Alzheimer's Disease plus Cerebrovascular Disease
}

\author{
Yong Kyun Kim ${ }^{a} \quad$ Kil-Byung Lim $^{b}$ Sang Chul Lee ${ }^{c}$ Jin-Woo Park ${ }^{d}$ \\ Hong-Jae Lee $^{b}$ Bum Sun Kwon ${ }^{d}$ Ho Jun Lee ${ }^{d}$ \\ a Department of Physical Medicine and Rehabilitation, Myongji Hospital, Kwandong \\ University College of Medicine, Goyang, ${ }^{b}$ Department of Physical Medicine and \\ Rehabilitation, Ilsan Paik Hospital, Inje University, Gimhae-si, ' Department of Physical \\ Medicine and Rehabilitation, Yonsei University College of Medicine, Seoul, and \\ dDepartment of Physical Medicine and Rehabilitation, Dongguk University College of \\ Medicine, Gyeongju-si, South Korea
}

\section{Key Words}

Alzheimer's disease with cerebrovascular disease - Rivastigmine patch · Activities of daily living

\begin{abstract}
Background/Aims: In dementia patients, a deficit in activities of daily living (ADL) is one of the main problems. Our objective was to assess ADL using the Korean Modified Barthel Index (K-MBI) in patients with Alzheimer's disease (AD) plus cerebrovascular disease (CVD) treated with a rivastigmine patch for 24 weeks in an open-label, observational study. Methods: 29 patients were enrolled who met the National Institute of Neurological and Communicative Disorders and Stroke/Alzheimer's Disease and Related Disorders Association (NINCDS/ ANDRA) criteria and had a score of 10-26 on the Korean version of the Mini-Mental State Examination (K-MMSE). After the rivastigmine patch had been applied for 24 weeks, changes in self-care activities were assessed using the K-MBI. Results: The average age of the patients was 62.8 years, and they had an average K-MMSE score of 16.2. Patients showed a mean improvement of 21.9 points, as compared with the baseline K-MBI score of $30.3(p<0.05)$. Significantly better outcomes were seen in secondary outcome variables, for example the K-MMSE and backward digit span. The most frequent adverse events were skin problems, such as itching sensation (10\%). Conclusion: In this multicenter, open-label, observational study, the rivastigmine patch was associated with improvements in ADL in patients with $A D$ plus CVD.


Kim et al.: Effects of a Rivastigmine Patch on Self-Care Activities in Patients with Alzheimer's Disease plus Cerebrovascular Disease

\section{Introduction}

Alzheimer's disease (AD) is clinically defined as a decline in cognition, a deterioration in the ability to perform activities of daily living (ADL), and having behavioral problems [1-3]. There is increasing evidence that $\mathrm{AD}$ frequently coexists with significant cerebrovascular disease (CVD) pathology [4]. Clinically, AD with CVD coexists in many dementia patients [5].

Patients with AD plus CVD need assistance in all aspects of daily living because of a cognitive decline, even though they have good muscle strength, coordination, and balance. The level of dependency of the patients is a measurement reflecting the level of severity of the disease which can be seen in ADL [6].

The current standard therapy for mild-to-moderate AD with CVD is cholinesterase inhibitors $[7,8]$. Rivastigmine patch is one of the cholinesterase inhibitors. In 2007, a transdermal patch formulation of rivastigmine became available in the United States for the treatment of mild-to-moderate AD $[9,10]$. Its efficacy and safety are already known $[11,12]$. Improvement in cognition could secondarily lead to improvement in ADL. However, previous reports do not show consistent results [5], which might be due to a low sensitivity to assess ADL [13].

Our objective was to assess ADL using the Korean Modified Barthel Index (K-MBI) in patients with AD plus CVD treated with a rivastigmine patch for 24 weeks.

\section{Methods}

\section{Subjects}

This open-label, single-arm study assesses the efficacy of a rivastigmine patch on ADL in patients with AD plus CVD using the K-MBI.

Patients with the following inclusion criteria were enrolled in the study: male or female individuals with a diagnosis of possible AD plus CVD according to the National Institute of Neurological and Communicative Disorders and Stroke/Alzheimer's Disease and Related Disorders Association (NINCDS/ADRDA) criteria [14]. Further inclusion criteria were subjects with a dementia onset after the age of 50 and a score of 10-26 on the Korean version of the Mini-Mental State Examination (K-MMSE).

Patients were excluded from the study if they had other neurologic or psychiatric disorders (schizophrenia, severe major depression), and if they were suspected of having been addicted to drugs or alcohol for the past 10 years. Patients with hepatic, renal, pulmonary, or cardiovascular disorders, such as bradycardia (pulse rate $<50$ ), sick sinus syndrome or any medical condition which would prevent them from completing the study, or patients who did not have the appropriate characteristics to be included in the study according to the investigator were excluded as well.

Fifty subjects were calculated on the basis of power calculation; the power was $80 \%$, with an alpha value of 0.05 , and the expected change in the values on the K-MBI after 24 weeks was $10 \pm 5$ in the pilot study.

At the first visit, subjects went through history taking, physical examination, evaluation of ADL by the K-MBI, of cognition by the K-MMSE and of language by the Korean version of the Western Aphasia Battery. After the subjects had decided to use the rivastigmine patch, those who agreed to give personal data on medical information were enrolled. Then, they were assigned to $5 \mathrm{~cm}^{2}$ of rivastigmine patch (delivering $4.6 \mathrm{mg} / 24 \mathrm{~h}$ ) for 4 weeks. After this titration period, the side effects, if any at all, were reported. Thereafter, the subjects were treated with $10 \mathrm{~cm}^{2}$ of rivastigmine patch (delivering $9.5 \mathrm{mg} / 24 \mathrm{~h}$ ) for another 20 weeks. Therefore, the total duration of the study was 24 weeks. After 24 weeks of applying a rivastigmine patch, ADL changes were reevaluated by using the K-MBI. 
Table 1. Characteristics of the subjects

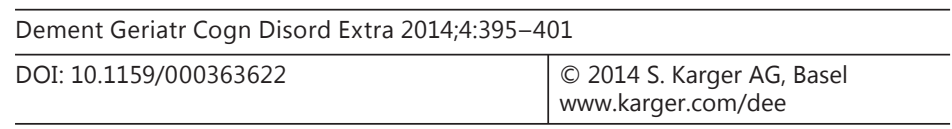

Kim et al.: Effects of a Rivastigmine Patch on Self-Care Activities in Patients with Alzheimer's Disease plus Cerebrovascular Disease

$\begin{array}{lc}\text { Age, years } & 62.8 \pm 11.4 \\ \text { Sex (female:male) } & 13: 16 \\ \text { Diagnosis, } \mathrm{n} & 29 \\ \text { Cerebral infarction, } \mathrm{n} & 14 \\ \text { Intracranial hemorrhage, } \mathrm{n} & 14 \\ \text { Hypoxic brain injury, } \mathrm{n} & 1\end{array}$

\section{Outcome Measures}

The primary scale used was the K-MBI. Each performance item is rated on this ordinal scale with a given number of points assigned to each level or ranking. The Barthel scale was introduced in 1965 and yields a score of 0-20. It is composed of 10 categories, which are bowel control, bladder control, grooming, toileting, transfer, mobility, dressing, climbing stairs, bathing, and feeding. The subjects were given a score for each category according to their independence level, where dependent $=0$, needs some help $=1$, and independent $=2$. The K-MBI was developed in 2007. It consists of personal hygiene, bathing, self-feeding, toileting, stair climbing, dressing, bowel control, bladder control, ambulation or wheelchair, and chair/bed transfer. The subjects were given a score for each item according to the independence level, where dependent $=0$, needs $75 \%$ assistance $=2$, needs $50 \%$ assistance $=5$, needs $25 \%$ assistance $=8$, and independent $=10$. The range of the score of the K-MBI is $0-$ 100 with a higher score implying more independence in ADL. The K-MBI is a valid and reliable method for measuring the functional status of the elderly (validity $=0.94$, reliability $=0.97$, and interrater agreement in 10 items $=0.82-1.0$ ). It has been used in many clinical studies. The patients were evaluated at baseline and after 24 weeks.

Secondary variables were assessed using 5 categories. The K-MMSE evaluated cognitive function, the Korean version of the Western Aphasia Battery was used for language abilities, the forward and backward digit span for attention deficit, the Neuropsychiatric Inventory Questionnaire for abnormal behavior, and the Controlled Oral Word Association Test for frontal executive function. Those were evaluated at baseline and 24 weeks after applying the rivastigmine patch.

\section{Statistical Analysis}

After analyzing the results of the primary and secondary variables, we performed a paired t test to assess the differences in the primary or secondary variables between baseline and 24 weeks. The analysis was performed with SPSS 12.0 (Statistical Package for the Social Science).

\section{Results}

Forty-seven patients with possible AD plus CVD were included in this study. Among those, 18 subjects were excluded. The most frequent reason for discontinuation was loss to follow-up ( $n=12,25.5 \%)$. Four subjects were discarded for complaining of itching sensation $(8.5 \%)$ on the site of applying the transdermal patch. One subject was excluded from this study due to an insurance issue because of an improvement in K-MMSE score. Unfortunately, 1 study could not be completed because the subject died. This leaves us with a total of 29 subjects who completed this study.

The average age of the study group was $62.8 \pm 11.4$ years; $44.8 \%$ were women (table 1 ). The baseline average K-MBI score was 30.3 points and the baseline mean MMSE score was 16.2 points. 
Kim et al.: Effects of a Rivastigmine Patch on Self-Care Activities in Patients with Alzheimer's Disease plus Cerebrovascular Disease

Fig. 1. a K-MBI. The baseline average K-MBI score was 30.3. A mean improvement of 21.9 was observed as compared with the baseline K-MBI score $(* \mathrm{p}<0.05)$. b Subscales of the K-MBI. There was a moderate improvement in several subscale scores of the $\mathrm{K}$ MBI, such as feeding, dressing, toileting, transfer, gait, bladder control and bowel control.
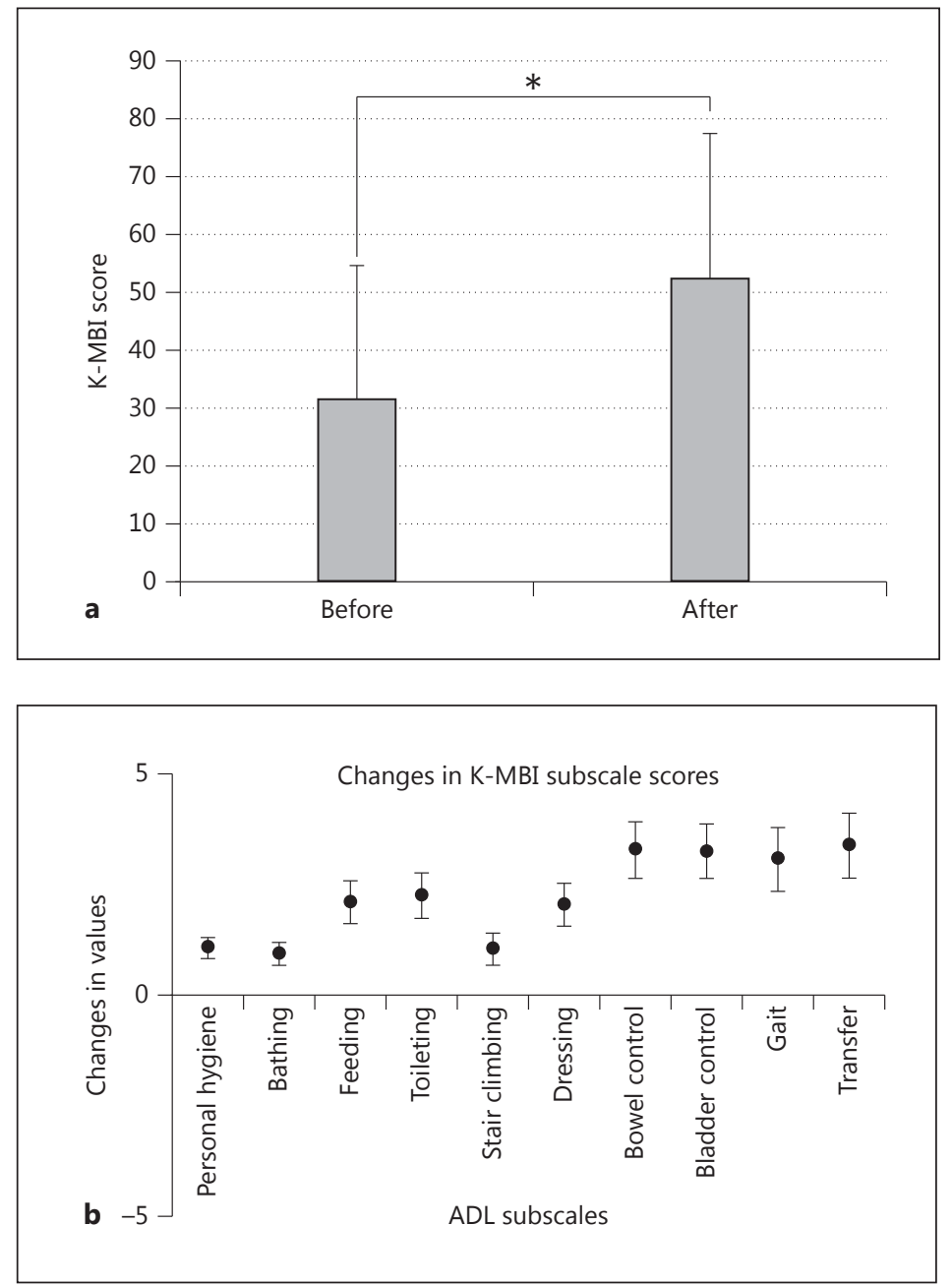

A primary efficacy variable was the mean change in the K-MBI score at 24 weeks, compared with the baseline score, in patients with AD plus CVD. Patients treated with a rivastigmine patch showed a mean improvement of 21.9 points $(p<0.05)$ (fig. 1a). On the subscale analysis of self-care activities, improvements were observed especially in eating, toileting, dressing, transfer, gait, bladder control, and bowel control (fig. 1b).

Significantly better outcomes were seen in secondary efficacy variables, for example, the K-MMSE and the backward digit span in the Seoul Neuropsychological Screening Battery. The mean K-MMSE score increased by 3.7 points ( $<<0.05$ ) (fig. 2). In the backward digit span test, the mean improvement was 0.9 points $(p<0.05)$ (fig. 3 ).

The most frequent adverse events were skin problems, such as itching on the site where the rivastigmine patch had been applied (about $8.5 \%$ of patients).

\section{Discussion}

The level of dependency in ADL of patients with AD plus CVD has an effect on the quality of life of both patients and caregivers $[1,6,15]$. This study showed that rivastigmine patches have positive effects on the self-care activities assessed by the K-MBI in patients with AD plus CVD. The result of the subscale analysis of the K-MBI scores, showing a beneficial effect of the 
Fig. 2. K-MMSE. The baseline average K-MMSE score was 16.2. A mean improvement of 3.7 was observed as compared with the baseline MMSE score ( $* \mathrm{p}<0.05)$.

Fig. 3. Backward digit span score. The baseline average score of the backward digit span test was 0.8 . A mean improvement of 0.9 was observed as compared with the baseline score $(* p<0.05)$.
Kim et al.: Effects of a Rivastigmine Patch on Self-Care Activities in Patients with Alzheimer's Disease plus Cerebrovascular Disease
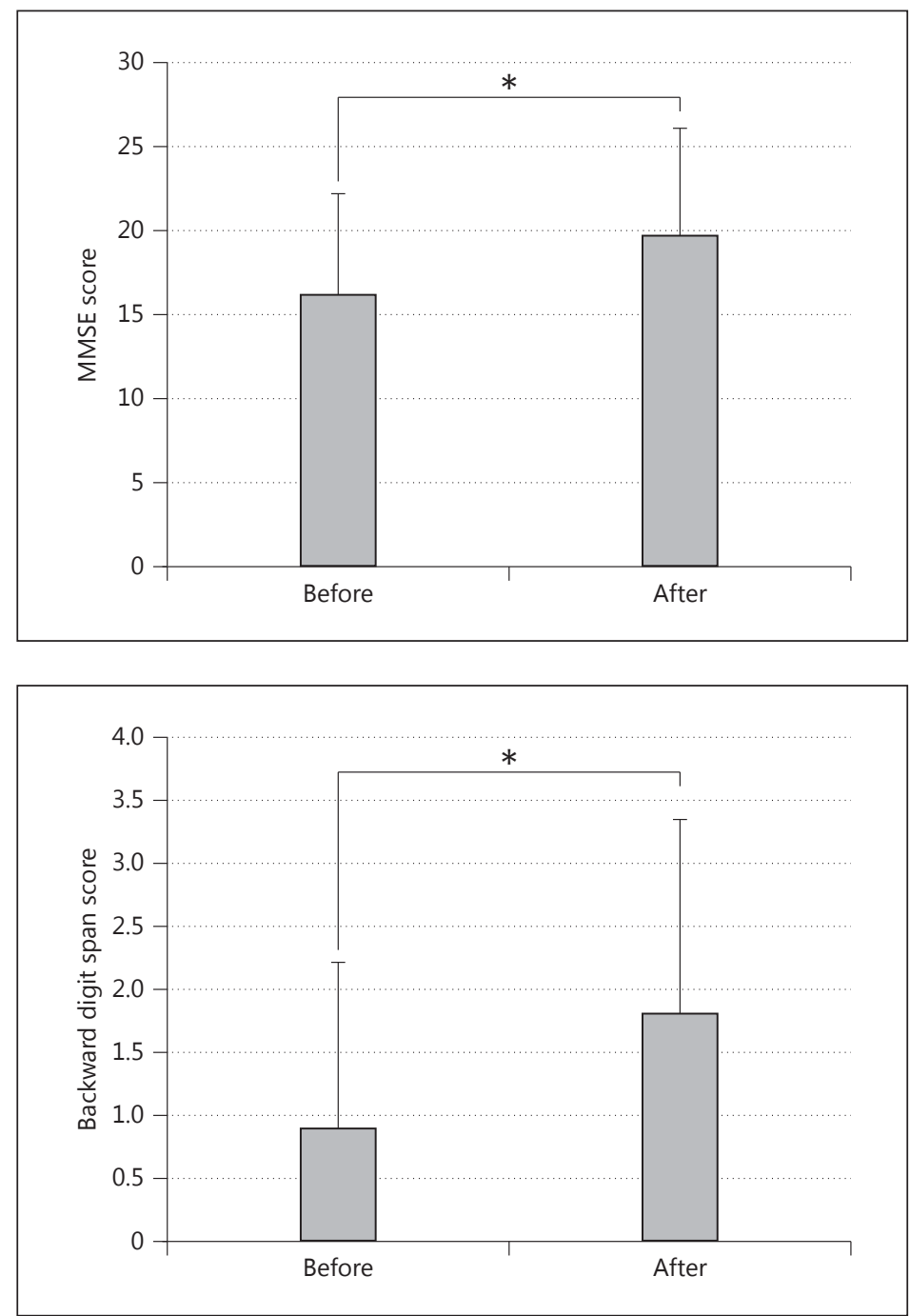

rivastigmine patch on the bowel control, bladder control, and dressing in patients with AD plus CVD, was compatible with a recent study [3].

In a previous study, ADL was assessed as a measure using the 23-item ADCS-ADL inventory [16]. The ADCS-ADL is a questionnaire with a possible score of $0-78$. Although the ADCS-ADL has many items (e.g. eating, walking, toileting), each category was included in only 2-4 other questionnaires for assessing ADL impairment levels. In this study, we chose to use the K-MBI to evaluate ADL. The K-MBI has 10 items and each category has 5 levels of assistance: 'unable to perform a task', 'maximal assistance', 'moderate assistance', 'minimal assistance', and 'full independent'. Thus, this evaluation tool is more sensitive for the assessment of ADL in patients with AD plus CVD.

With respect to secondary outcome measures, at week 24, patients treated with the rivastigmine patch showed improvement in K-MMSE scores. These data are similar to those in a previous study [17]. Among the cognitive domains of the K-MMSE, improvements were observed in the attention and visuospatial function domain. This finding might be associated with a significant improvement in the backward digit span test of the Seoul Neuropsychological Screening Battery after 24 weeks of rivastigmine patch treatment. We report that the rivastigmine patch might be working more effectively on the visuospatial function in $A D$ 
patients with CVD. A possible explanation for this is that the visuospatial function is related to the posterior parietal cortex of the right hemisphere of the brain. This area has a lot of connections to the subcortical area such as basal ganglia, thalamus, and the limbic system. Therefore, rivastigmine treatment might lead to a more efficient connection between the right parietal cortex and the subcortical area. This interesting finding is compatible with the results of a recent study showing that rivastigmine treatment may be useful to accelerate the recovery of unilateral spatial neglect [18].

Previous studies reported placebo or untreated mean declines on the MMSE of approximately 2-3.5 points after 1 year, and of approximately 5.5-6.5 points after 2 years in patients with baseline MMSE scores of 10-26 [19,20]. Also, annual placebo declines of approximately 11.5 points on the ADCS-ADL have been reported [21]. In our study, patients treated with the rivastigmine patch showed improvement in the K-MMSE and K-MBI scores at 24 weeks of approximately 4 and 22 points on average, respectively. One of the possible reasons for this might be the fact that most of our patients were at 4 months after stroke at the time of enrollment in this study.

About $8.5 \%$ of the study population discontinued the trial because of application site reactions, such as itching sensation or redness. These findings were consistent with a previous 6-month placebo-controlled trial [22]. However, gastrointestinal symptoms such as nausea, vomiting, and diarrhea were rare.

Several potential limitations of this study include: the open-label nature of the observational study design or the lack of direct concurrent comparison with a placebo control group, the relatively small sample size, and the fact that it was not a double-blind randomized controlled study.

Therefore, in the future, a double-blind randomized controlled study must be performed to clarify the role of rivastigmine in promoting functional recovery and improving cognition in patients with AD plus CVD for a long-term period.

\section{Conclusion}

In this multicenter, open-label, 24-week observational study, the rivastigmine patch led to improvements in the K-MBI scores in patients with AD plus CVD.

\section{Acknowledgement}

Novartis Pharmaceuticals supported the study.

\section{Disclosure Statement}

There are no conflicts of interest.

\section{References}

1 Alva G, Grossberg GT, Schmitt FA, Meng X, Olin JT: Efficacy of rivastigmine transdermal patch on activities of daily living: item responder analyses. Int J Geriatr Psychiatry 2011;26:356-363.

-2 Cummings JL, Koumaras B, Chen M, Mirski D: Effects of rivastigmine treatment on the neuropsychiatric and behavioral disturbances of nursing home residents with moderate to severe probable Alzheimer's disease: a 26-week, multicenter, open-label study. Am J Geriatr Pharmacother 2005;3:137-148. 
Kim et al.: Effects of a Rivastigmine Patch on Self-Care Activities in Patients with Alzheimer's Disease plus Cerebrovascular Disease

-3 Grossberg G, Meng X, Olin JT: Impact of rivastigmine patch and capsules on activities of daily living in Alzheimer's disease. Am J Alzheimers Dis Other Demen 2011;26:65-71.

4 Jellinger KA: The enigma of mixed dementia. Alzheimers Dement 2007;3:40-53.

5 Erkinjuntti T, Gauthier S, Bullock R, Kurz A, Hammond G, Schwalen S, Zhu Y, Brashear R: Galantamine treatment in Alzheimer's disease with cerebrovascular disease: responder analyses from a randomized, controlled trial (GAL-INT-6). J Psychopharmacol 2008;22:761-768.

-6 Andersen CK, Wittrup-Jensen KU, Lolk A, Andersen K, Kragh-Sorensen P: Ability to perform activities of daily living is the main factor affecting quality of life in patients with dementia. Health Qual Life Outcomes 2004; $2: 52$.

7 Bullock R, Erkinjuntti T, Lilienfeld S: Management of patients with Alzheimer's disease plus cerebrovascular disease: 12-month treatment with galantamine. Dement Geriatr Cogn Disord 2004;17:29-34.

-8 Small G, Erkinjuntti T, Kurz A, Lilienfeld S: Galantamine in the treatment of cognitive decline in patients with vascular dementia or Alzheimer's disease with cerebrovascular disease. CNS Drugs 2003;17:905-914.

-9 Moretti R, Torre P, Antonello RM, Cazzato G, Bava A: Rivastigmine in subcortical vascular dementia: an open 22-month study. J Neurol Sci 2002;203-204:141-146.

10 Vincent S, Lane R: Rivastigmine in vascular dementia. Int Psychogeriatr 2003;15(suppl 1):201-205.

11 Farlow MR, Lilly ML: Rivastigmine: an open-label, observational study of safety and effectiveness in treating patients with Alzheimer's disease for up to 5 years. BMC Geriatr 2005;5:3.

12 Kumar V, Anand R, Messina J, Hartman R, Veach J: An efficacy and safety analysis of Exelon in Alzheimer's disease patients with concurrent vascular risk factors. Eur J Neurol 2000;7:159-169.

13 Ylikoski R, Jokinen H, Andersen P, Salonen O, Madureira S, Ferro J, Barkhof F, van der Flier W, Schmidt R, Fazekas F, et al: Comparison of the Alzheimer's Disease Assessment Scale Cognitive Subscale and the Vascular Dementia Assessment Scale in differentiating elderly individuals with different degrees of white matter changes. The LADIS Study. Dement Geriatr Cogn Disord 2007;24:73-81.

-14 McKhann G, Drachman D, Folstein M, Katzman R, Price D, Stadlan EM: Clinical diagnosis of Alzheimer's disease: report of the NINCDS-ADRDA Work Group under the auspices of Department of Health and Human Services Task Force on Alzheimer's Disease. Neurology 1984;34:939-944.

15 Kurz X, Scuvee-Moreau J, Rive B, Dresse A: A new approach to the qualitative evaluation of functional disability in dementia. Int J Geriatr Psychiatry 2003;18:1050-1055.

16 Galasko D, Bennett D, Sano M, Ernesto C, Thomas R, Grundman M, Ferris S: An inventory to assess activities of daily living for clinical trials in Alzheimer's disease. The Alzheimer's Disease Cooperative Study. Alzheimer Dis Assoc Disord 1997;11(suppl 2):S33-S39.

17 Articus K, Baier M, Tracik F, Kühn F, Preuss UW, Kurz A: A 24-week, multicentre, open evaluation of the clinical effectiveness of the rivastigmine patch in patients with probable Alzheimer's disease. Int J Clin Pract 2011;65: 790-796.

18 Paolucci S, Bureca I, Multari M, Nocentini U, Matano A: An open-label pilot study of the use of rivastigmine to promote functional recovery in patients with unilateral spatial neglect due to first ischemic stroke. Funct Neurol 2010;25:195-200.

19 Bullock R, Touchon J, Bergman H, Gambina G, He Y, Rapatz G, Nagel J, Lane R: Rivastigmine and donepezil treatment in moderate to moderately-severe Alzheimer's disease over a 2-year period. Curr Med Res Opin 2005;21:1317-1327.

20 Small GW, Kaufer D, Mendiondo MS, Quarg P, Spiegel R: Cognitive performance in Alzheimer's disease patients receiving rivastigmine for up to 5 years. Int J Clin Pract 2005;59:473-477.

-21 Aisen PS, Schafer KA, Grundman M, Pfeiffer E, Sano M, Davis KL, Farlow MR, Jin S, Thomas RG, Thal LJ: Effects of rofecoxib or naproxen versus placebo on Alzheimer disease progression: a randomized controlled trial. JAMA 2003;289:2819-2826.

22 Winblad B, Kawata AK, Beusterien KM, Thomas SK, Wimo A, Lane R, Fillit H, Blesa R: Caregiver preference for rivastigmine patch relative to capsules for treatment of probable Alzheimer's disease. Int J Geriatr Psychiatry 2007;22:485-491. 